\title{
Density Matrix Renormalization Group Study of the Spin 1/2 Heisenberg Ladder with Antiferromagnetic Legs and Ferromagnetic Rungs
}

\author{
Kazuo HIDA \\ Department of Physics, Faculty of Science, \\ Saitama University, Urawa, Saitama 338 \\ fi (Received )
}

\begin{abstract}
The ground state and low lying excitation of the spin 1/2 Heisenberg ladder with antiferromagnetic leg $(J)$ and ferromagnetic rung $(-\lambda J, \lambda>0)$ interaction is studied by means of the density matrix renormalization group method. It is found that the state remains in the Haldane phase even for small $\lambda \sim 0.02$ suggesting the continuous transition to the gapless phase at $\lambda=0$. The critical behavior for small $\lambda$ is studied by the finite size scaling analysis. The result is consistent with the recent field theoretical prediction.
\end{abstract}

Keywords: Heisenberg ladder, Haldane gap, Density matrix renormalization group e-mail: hida@th.phy.saitama-u.ac.jp 


\section{INTRODUCTION}

Recently, the spin-1/2 Heisenberg ladder model has been studied extensively [1 9,?,11 17 motivated by its relevance to high $T_{c}$ superconductivity and Haldane gap problem. [18 21] The former is concerned with the fully antiferromagnetic ladder, while the latter is related to the ladder with ferromagnetic rung and antiferromagnetic leg interactions. In the present paper, we concentrate on the latter model.

Several years ago, the present author studied this model using the projctor Monte Carlo method. [1] It has been further studied by the non-linear $\sigma$ model, [2] the bosonization method [3, 11-13] and exact diagonalization. [14] For small interchain coupling, however, their conclusions are still controversial. The bosonization calculation by Watanabe, Nomura and Takada [3] support the Kosterlitz-Thouless (KT) type behavior with the energy gap proportional to $\exp (-$ const. $/ \sqrt{\lambda})$, and the nonlinear $\sigma$ model analysis [2] predicts the possiblity of the KT type transition to the gapless state at nonzero positive $\lambda$. Although the projector Monte Carlo simulation [1] seems to support one of these two kinds of possibility, the data are rather scattered. On the other hand, the non-Abelian bosonization studies by Strong and Millis [11,12] suggest the continuous transition at $\lambda=0$ where energy gap is proportional to $\lambda$. Using the same method, Totsuka and Suzuki [13 predict the presence of the logarithmic correction to the gap as $\lambda(\ln (1 / \lambda))^{1 / 2}$. The exact diagonalization study of this model has been also performed by Watanabe. [14] Nishiyama et al. [15] and Terai [16] made the perturbational approach from $\lambda=0$ for the finite size system. The density matrix renormalization group (DMRG) 22,23] study is also carried out by Narushima et al. [17] These works support the continuous transition at $\lambda=0$ with the energy gap exponent $\nu \simeq 1$ with possible logarithmic correction. It is the purpose of the present work to investigete this problem further analyzing the DMRG data by the finite size scaling method.

In the next section, we explain the model and the method of the DMRG calculation. The results are analyzed in sections 3 and 4 . The last section is devoted to summary and discussion. 


\section{HEISENBERG LADDER}

We consider the spin 1/2 Heisenberg ladder consisting of two coupled Heisnberg chains represented by the following Hamiltonian $H$.

$$
H=2 J \sum_{i=1}^{N}\left[\boldsymbol{S}_{i}^{A} \boldsymbol{S}_{i+1}^{A}+\boldsymbol{S}_{i}^{B} \boldsymbol{S}_{i+1}^{B}-\lambda \boldsymbol{S}_{i}^{A} \boldsymbol{S}_{i}^{B}\right], \quad(J, \lambda>0)
$$

where $\boldsymbol{S}_{i}^{\alpha}(\alpha=A$ or $B)$ is the spin operator with spin $1 / 2$. The length of the ladder is denoted by $N$. For $\lambda \rightarrow \infty$, the spins $\boldsymbol{S}_{i}^{A}$ and $\boldsymbol{S}_{i}^{B}$ form a local triplet and this model reduces to the spin-1 Heisenberg chain. On the other hand, this model is decoupled into two spin $1 / 2$ antiferromagnetic Heisenberg chains for $\lambda=0$.

In the DMRG transform, [22,23] the number $m$ of the states retained in the left and right half systems ranges from 80 to 140 . We use the mixed algorithm of finite size and infinite size method. Namely, during the increase of the system size by the infinite size algorithm, we sometimes improve the accuracy by moving the boundary between the left and right half systems until the eigenvalues converge keeping the system size constant. In order to save the computational time, however, we only move the boundary among the sites which are added after the last finite size iteration. The data are taken only for the sizes for which the finite size iteration is made. For $m=140$, only the infinite size algorithm is used unless specifically mentioned. On each step of the infinite size iteration, 4 spins are added. In the following, we only consider the case of even $N$.

In the calculation of the low lying excitation energy eigenvalues, we have constructed the density matrices of the left and right half systems from the lowest 3 states of the whole system with fixed $S_{z}^{\text {tot }}(=z$-component of the total spin). Let us denote the energy eigenvalue

of the $n$-th excited state with $S_{z}^{\text {tot }}$ by $E\left(n, S_{z}^{\text {tot }}\right)$ where $n=0$ corresponds to the ground state with given value of $S_{z}^{\text {tot }}$. For large $N$, the calculated energy gap depend on $m$. We assume that the finite $m$ correction behaves as $1 / m^{2}$ for large $m$ as pointed out by Narushima et al. [17] Taking this into account, we tried two different ways of $m$-extrapolation. First, the data are extrapolated from $m=100,120$ and 140 linearly to $1 / m^{2}$ by the least squares fit. 
Secondly, $1 / m^{3}$-correction is included using the data for $m=80,100,120$ and 140 . The data points are the average of these two extrapolated values and the error bars are estimated from the difference between them.

\section{KENNEDY TRIPLET}

One of the most remarkable features of the Haldane phase is the presence of the four-fold quasi-degeneracy of the ground state and low lying states 21] in the open boundary system. In analogy with the $S=1$ antiferromagnetic Heisenberg chain, the ground state is a singlet and the three lowest excited states form a triplet called Kennedy triplet [21] if the ladder length $N$ is even.

Fig. 1 shows the semi-log plot of the size dependence of the excitation energy $\Delta_{K}=$ $E(1,0)-E(0,0)$ of the lowest excited states with $S_{z}^{\text {tot }}=0$ extrapolated to $m \rightarrow \infty$. The

convergence of the numerical diagonalization procedure becomes poor for some points due to the reason explained in the next section. These data are excluded from the analysis. For large $N$, the excitation energy vanishes exponentially with the increase of the system size within the error bars confirming that the system is in the Haldane phase down to $\lambda \sim 0.02$. This implies that the continuous transition to the gapless phase takes place at $\lambda=0$. The same conclusion is also obtained by Narushima et al. [17]

\section{HALDANE GAP}

Taking into account the presence of the Kennedy triplet, the Haldane gap may be estimated by extrapolating the difference $\Delta E_{0}=E(2,0)-E(1,0)$ [14 to $N \rightarrow \infty$. The system size dependence of $\Delta E_{0}$ is shown in Fig. 2 for $\lambda=0.02,0.05,0.1,0.15$ and 0.2 and $4 \leq N \leq 102$. The second excitation gap $\Delta E_{1}=E(3,0)-E(1,0)$ is also shown for small systems $(N \leq 12)$ which can be diagonalized exactly. From this figure, we find that the gap $\Delta E_{0}$ has a maximum at $N=N_{\max }(\lambda)$ which increases with the decrease of $\lambda$. 
This maximum results from the level crossing which takes place in the finite size system at $\lambda=\lambda_{\mathrm{c}}(N)$. This level crossing is already pointed out by Watanabe [14 and the physical picture of these excitations are also given. Roughly speaking, we may regard $N_{\max }(\lambda)$ as the inverse function of $\lambda_{c}(N)$, although this is not well-defined in the mathematical sense because $N$ is a discrete variable.

The gap $\Delta E_{0}$ for $N>N_{\max }(\lambda)$ continue to the second excitation gap $\Delta E_{1}$ for $N<$ $N_{\max }(\lambda)$ as shown in Fig. 2. Let us define the gap $\Delta_{\mathrm{q}}$ by $\Delta E_{0}$ for $N>N_{\max }$ and $\Delta E_{1}$ for $N<N_{\max }$, because this state corresponds to the quintuplet excitation. [14 In the thermodynamic limit $N \rightarrow \infty, \Delta_{\mathrm{q}}$ corresponds to the Haldane gap. Similarly, second triplet excitation gap $\Delta_{\mathrm{t}}$ is defined by $\Delta E_{1}$ for $N>N_{\max }$ and $\Delta E_{0}$ for $N<N_{\max }$, although we did not calculate $\Delta E_{1}$ for $N>N_{\max }$ because the accuracy of the higher excitation energy becomes worse in the DMRG method. Nevertheless, we have shown $\Delta_{\mathrm{t}}$ for $\lambda=0.05$ for large $N$. These eigenvalues are obtained as the second excited state in the DMRG caluculation. However, we interprete these values as $\Delta_{\mathrm{t}}$ instead of $\Delta_{\mathrm{q}}$, because it is on the line smoothly extrapolated from $\Delta_{t}$. We expect that this occurs because the states important for the quintuplet states are already discarded during the iteration for $N<N_{\max }$. Due to the same reason, the gap $\Delta_{\mathrm{q}}$ is less accurate than $\Delta_{\mathrm{t}}$ for large $N$. This makes difficult to determine the precise value of the exponent $\nu$ for $\Delta_{\mathrm{q}}$.

We therefore start with the analysis of the system size dependence of $\Delta_{\mathrm{t}}$ by the finite size scaling method. In this analysis, we use only the data obtained after the finite size iteration even for $m=140$. According to Strong and Millis [11,12 and Totsuka and Suzuki, 13 the field theoretical calculation using the non-Abelian bosonization technique yields $\nu=1$. Totsuka and Suzuki [13] also noted the presence of the logarithmic correction to the gap as $\lambda(\ln (1 / \lambda))^{1 / 2}$. Expecting the similar behavior for $\Delta_{t}$, let us assume the finite size scaling formula for $\Delta_{\mathrm{t}}$ as,

$$
N \Delta_{\mathrm{t}}(N)=f\left(N(\ln N)^{\alpha} \lambda^{\nu}\right)
$$

This scaling formula is also employed by Terai. [16] As discussed by Spronken et al. [24], this 
scaling relation implies that the gap $\Delta_{\mathrm{t}}$ of the infinite system behaves as $\Delta_{\mathrm{t}} \sim \lambda^{\nu}(\ln (1 / \lambda))^{\alpha}$. We have tried to fix both $\alpha$ and $\nu$ by the least squares fit to the universal curve $f$ using the data for $\lambda=0.02,0.05,0.1$ and 0.2 and $4 \leq N \leq 102$. The data with large error bars $\left(\delta\left(N \Delta_{\mathrm{t}}\right) \gtrsim 1\right)$ are excluded from the analysis. However, the least squares deviation is rather insensitive to the value of $\alpha$ while the optimum value of $\nu$ remains around unity irrespective of the choice of $\alpha$. For example, Fig. 3 shows the plot with $\nu=0.88$ and $\alpha=0$ which is the optimum choice with $\alpha=0$. Fig. 4 shows the fit with $\alpha=0.4$ and $\nu=1$ which is the optimum choice with $\nu=1$. In both cases, the data $N \Delta_{\mathrm{t}}$ excellently fits the single universal curve $f(x)\left(x=N(\ln N)^{\alpha} \lambda^{\nu}\right)$. Although the available value of $x$ for $\Delta_{\mathrm{t}}$ is limited, $f(x)$ behaves linearly for large $x$ within the present data. The scaling plot of $\Delta_{\mathrm{q}}$ is also shown. For large $N$, it also follows another universal curve which also tends to a linear function of $x$ with the same scaling exponent fairly well. This suggests that the critical behavior of $\Delta_{\mathrm{q}}$ is also governed by the same exponents as those for $\Delta_{\mathrm{t}}$.

We can obtain almost equally good fit using other sets of values of $\alpha$ and $\nu$ as far as $\nu \sim 1$ both for $\Delta_{\mathrm{t}}$ and $\Delta_{\mathrm{q}}$. This clearly excludes the possibility of KT type behavior. However, this in turn means that it is difficult to determine the value of $\alpha$ solely from the numerical data. Therefore, taking into account the estimations from other works [1] [13, 15] 17] for the exponents of the Haldane gap and assuming that $\Delta_{\mathrm{q}}$ and $\Delta_{\mathrm{t}}$ has the same exponents as discussed above, we fix the value of $\nu$ to unity and search for the optimum value of $\alpha$. In this way, we find $\alpha=0.4 \pm 0.07$. The error of $\alpha$ is estimated from that of $\Delta_{\mathrm{t}}$ due to $m$-extrapolation. This value is close to the analytical estimation $\alpha=0.5$ by Totsuka and Suzuki [13] and perturbational estimation of [16].

The finite size scaling plot in Fig. 4 also suggests that the level crossing point is also scale invariant as $\lambda_{c}(N) N(\ln N)^{\alpha}=$ const. $\sim 5.2$. This implies that $\lambda_{c}$ tends to 0 as $N \rightarrow \infty$, confirming that the gap $\Delta_{\mathrm{q}}$ remains the lowest gap down to $\lambda=0$ in the thermodynamic limit. 


\section{SUMMARY AND DISCUSSION.}

We have studied the low energy excitation of the spin-1/2 Heisenberg ladder with ferromagnetic rungs and antiferromagnetic legs using the DMRG method. It is found that the ground state remains in the Haldane phase down to $\lambda=0.02$ suggesting the continous transition to the gapless phase at $\lambda=0$. The exponent $\nu$ of the second triplet gap $\Delta_{\mathrm{t}}$ is estimated to be around unity using the finite size scaling analysis. Fixing $\nu=1$, we find the logarithmic correction as $\Delta_{\mathrm{t}} \sim \lambda(\ln (1 / \lambda))^{\alpha}$ with $\alpha=0.4 \pm 0.07$. Although the numerically obtained value of $\Delta_{\mathrm{q}}$ is not enough accurate to determine the exponents independently from $\Delta_{\mathrm{t}}$, it is expected $\Delta_{\mathrm{q}}$ also shows the same critical behavior taking into account the fact that $\Delta_{\mathrm{q}}$ also fits to the universal curve fairly well with the same scaling within the error bars. It is also shown that $\Delta_{\mathrm{q}}$ remains the lowest gap (Haldane gap) down to $\lambda \rightarrow 0$ in the thermodynamic limit.

Several years ago, the present author proposed the possibility of the transition at finite $\lambda$ based on the projector Monte Carlo simulation [1] and the mapping onto the nonlinear $\sigma$ model. [2] However, the Monte Carlo data are rather scattered and the nonlinear $\sigma$ model analysis is based on the semiclassical approximation and the artificially introduced anisotropy. Therefore, we believe the present result is much more reliable. Actually, the non-Abelian bosonization analysis employed by Strong and Millis [11,12] and Totsuka and Suzuki, [13 which leads to the results consistent with our calculation, may be regarded as the refined version of the nonlinear $\sigma$ model analysis suited for the isotropic case.

\section{ACKNOWLEDGMENTS}

The author is indebted to H. Nishimori for TITPACK ver.2 for the diagonalization of spin $1 / 2$ system. He also thanks N. Hatano, A. Terai and H. Watanabe for comments and discussion. This work is partly supported by the Grand-in-Aid for Scientific Research from the Ministry of Education, Science and Culture. The numerical calculation has been 
performed using the FACOM VPP500 at the Supercomputer Center, Institute for Solid State Physics, University of Tokyo and the HITAC S820/15 at the Information Processing Center, Saitama University. 


\section{REFERENCES}

fi

fi

fi

fi

fi [5]T. Barnes, E. Dagotto, J. Riera and E.S. Swanson: Phys. Rev. B47 (1993) 3196.

fi [6]Sudha Gopalan, T.M. Rice and M. Sigrist: Phys. Rev. B49 (1994) 8901.

fi

fi

fi

fi [10]S. R. White, R. M. Noack and D. J. Scalapino: Phys. Rev. Lett. 73886 (1994).

fi

fi

fi

fi

[1]K. Hida: J. Phys. Soc. Jpn. 60 (1991) 1347.

[2]K. Hida: J. Phys. Soc. Jpn. 60 (1991) 1939.

[3]H. Watanabe, K. Nomura and S. Takada: J. Phys. Soc. Jpn. 62 (1993) 2845.

[4]E. Dagotto, J. Riera and D. Scalapino: Phys. Rev. B45 (1992) 5744.

[7]M. Azzouz, L. Chen and S. Moukouri: Phys. Rev. B50 (1994) 6233.

[8]T. Barnes and J. Riera: Phys. Rev. B50 (1994) 6817.

[9]M. Troyer, H. Tsunetsugu and D. Würtz: Phys. Rev. B50 13515 (1994).

[11]S. P. Strong and A. J. Millis: Phys. Rev. Lett. 692419 (1992).

[12]S. P. Strong and A. J. Millis: Phys. Rev. B50 9911 (1994).

[13]K. Totsuka and M. Suzuki: J. Phys. Condens. Matter 76079 (1995).

[14]H. Watanabe: Phys. Rev. B50 13442 (1994).

[15]Y. Nishiyama, N. Hatano and M. Suzuki: J. Phys.Soc. Jpn 641967 (1995).

[16]A. Terai: private communication.

[17]T. Narushima, T. Nakamura and S. Takada: preprint (1995).

[18]F.D.M. Haldane: Phys. Lett. 93A 464 (1983).

[19]F.D.M. Haldane: Phys. Rev. Lett. 501153 (1983).

[20]I. Affleck: J. Phys. Condens. Matter. 13047 (1989) and references therein. 
fi

fi

fi

fi
[21]T. Kennedy: J. Phys. Condens. Matter. 2 (1990) 5737.

[22]S. R. White: Phys. Rev. Lett. 692863 (1992).

[23]S. R. White: Phys. Rev. B48 10345 (1993).

[24]G. Spronken, B. Fourcade and Y. Lépine: Phys. Rev. B33 1886 (1986). 


\section{FIGURES}

FIG. 1. The system size dependence of the energy gap $\Delta_{K}$ between the ground state and the Kennedy triplet for $\lambda=0.02,0.05,0.1,0.15$ and 0.2 .

FIG. 2. The system size dependence of the energy gap $\Delta E_{0}$ (open symbols) and $\Delta E_{1}$ (filled symbols) for $\lambda=0.02,0.05,0.1,0.15$ and 0.2 .

FIG. 3. The finite size scaling plot with $\nu=0.88$ of the energy gap $\Delta_{\mathrm{t}}$ (open symbols) and $\Delta_{\mathrm{q}}$ (filled symbols) for $\lambda=0.02,0.05,0.1,0.15$ and 0.2 .

FIG. 4. The finite size scaling plot with $\nu=1$ and $\alpha=0.4$ of the energy gap $\Delta_{\mathrm{t}}$ (open symbols) and $\Delta_{\mathrm{q}}$ (filled symbols) for $\lambda=0.02,0.05,0.1,0.15$ and 0.2 . 